\title{
Zoledronic Acid after Treatment with Denosumab is Associated with Bone Loss within 1 Year
}

\author{
Tarun Kadaru', Amal Shibli-Rahhal ${ }^{2}$ \\ 'Department of Internal Medicine, University of Texas Southwestern, Dallas, TX; \\ ${ }^{2}$ Department of Internal Medicine, University of lowa Carver College of Medicine, lowa City, IA, USA
}

Corresponding author

Amal Shibli-Rahhal

Department of Internal Medicine, University of lowa Carver College of Medicine, 200 Hawkins Drive, Endocrinology Department Room 400 GH, lowa City, IA 52242, USA

Tel: +1-319-353-7812

Fax: $+1-319-353-7850$

E-mail: amal-rahhal@uiowa.edu

Received: November 28, 2020

Revised: December 19, 2020

Accepted: December 28, 2020
Background: Bone mineral density (BMD) declines when zoledronic acid (ZA) is administered. This case series describes the patterns of change in BMD when 1 or 2 doses of ZA are administered after denosumab. Methods: Twelve patients who received at least 2 doses of denosumab followed by at least 1 dose of ZA and who had a dual energy X-ray absorptiometry (DXA) scan at the end of denosumab and 1 year after the first dose of ZA were included. We excluded patients with bone cancer or conditions affecting bone metabolism, including hyperparathyroidism, rickets, osteogenesis imperfecta, rheumatologic disorders, fibrous dysplasia, Paget's disease of bone, untreated hyperthyroidism, chronic kidney disease, liver cirrhosis, malabsorption, ongoing corticosteroid therapy, and aromatase inhibitor use. Results: There was a significant decline in BMD at the femoral neck within 1 year of the first ZA dose and a non-significant downward trend in the hip and lumbar spine. This trend was more severe in patients with osteoporosis at the time of drug transition. No increase in clinical vertebral fractures was observed. BMD seemed to stabilize in a smaller number of patients who received a second dose of ZA and had a DXA scan 1 year later. Conclusions: A single dose of ZA administered approximately 6 months after denosumab leads to some BMD loss, mostly within 1 year of ZA administration, particularly in patients with osteoporosis at the time of denosumab discontinuation.

Key Words: Bone density · Denosumab $\cdot$ Zoledronic acid

\section{INTRODUCTION}

Denosumab is a monoclonal antibody that mimics the action of endogenous osteoprotegerin by binding to and preventing the action of the: receptor activator of nuclear factor-KB ligand (RANKL), thereby inhibiting osteoclasts and preventing bone resorption.[1,2] Denosumab is a potent antiresorptive agent, leading to significant increases in bone mineral density (BMD) and fracture prevention.[3] However, acute discontinuation of denosumab leads to a rebound rapid increase in bone turnover with a large bone loss [4-6] and in some cases is associated with an increase in the risk of vertebral compression fractures.[7-13] This phenomenon is believed to be due to a compensatory upregulation of the RANK receptors on osteoclast precursors while in the presence of denosumab.[4] To avoid this rebound increase in bone resorption and the associated bone loss, "consolidation therapy" has been recommended. This approach entails transitioning patients

\section{Mineral Research}

This is an Open Access article distributed under the terms of the Creative Commons Attribution Non-Commercial License (https://creativecommons.org/licenses/by-nc/4.0/ which permits unrestricted non-commercial use, distribution, and reproduction in any medium, provided the origina work is properly cited.

\section{KSBMR}


from denosumab to a bisphosphonate prior to complete discontinuation of antiresorptive therapy, $[13,14]$ and zoledronic acid (ZA) became one of the drugs commonly used in this setting. However, the use of ZA after denosumab does not seem to fully prevent the bone loss that follows discontinuation of denosumab. In a recent retrospective study by Everts-Graber et al. [15], patients previously treated with denosumab for 2 to 5 years followed by a single infusion of ZA experienced some loss of BMD on a dual energy X-ray absorptiometry (DXA) scan 12 to 42 months later. However, only patients who had achieved osteopenia while on denosumab were transitioned to ZA in this study, limiting those findings to a select group of patients with a rather robust response to denosumab.

In our clinical practice, we follow a protocol that attempts to limit total antiresorptive therapy (denosumab+consolidation with ZA) to no more than 5 to 6 years. To transition to $Z A$, patients should have had stable or improved BMD on denosumab for at least 1 year, but we do not aim to achieve osteopenia prior to initiating consolidation therapy. We routinely evaluate patient response on DXA around 12 months after ZA administration. In this case series, we describe changes in BMD and prevalence of clinical fractures 1 year after transitioning from denosumab to ZA in patients with both osteopenia and osteoporosis at the end of denosumab therapy. We also report observations on BMD changes after a second dose of ZA was given a year later to a smaller subgroup of patients.

\section{METHODS}

\section{Data sources and patients}

This is a retrospective case series of patients with osteoporosis treated with denosumab followed by ZA in the Endocrinology Clinic at an academic medical center. We used the electronic medical records (EMR; Epic 2018 version UI 2; Epic Systems Corp., Verona, WI, USA) to identify patients treated with denosumab followed by ZA between January 1, 2012 (the year denosumab was approved for clinical use in the USA) and June 30,2020. Eligible patients met the following inclusion criteria: (1) received at least 2 doses of denosumab; (2) followed by at least 1 dose of $Z A$; (3) underwent DXA at the end of their treatment course with denosumab and approximately 1 year after the first dose of ZA. Patients were excluded if they had bone cancer or oth- er conditions affecting bone metabolism including hyperparathyroidism, rickets, osteogenesis imperfecta, rheumatologic disorders, fibrous dysplasia of the bone, Paget's disease of the bone, untreated hyperthyroidism, chronic kidney disease, liver cirrhosis, malabsorptive conditions (such as inflammatory bowel disease or celiac disease), and ongoing glucocorticoid or aromatase inhibitor use.

The EMR contains complete demographic, clinical, laboratory, and medication data for all patients seen at our medical center.

\section{Data elements}

Key data elements included patient demographics, height, weight, body mass index (BMI), medical comorbidities, smoking status, alcohol consumption history, DXA measurements and date of each measurement, number of denosumab doses and date of each dose, and number of post-denosumab ZA doses and date of each dose. All doses of denosumab and ZA were administered at our center and dates of administration were extracted from the patients'EMR.

We also recorded basic laboratory results at the initiation of denosumab, including serum concentrations of calcium, creatinine, parathyroid hormone (PTH), and 25-hydroxy-vitamin D (25[OH]D). Only laboratory values obtained at the core laboratory at our medical center were recorded and used in this analysis to eliminate the bias that could be introduced by the variability between different assays. Calcium and creatinine were determined using Roche Modular P analyzers (Hoffmann-La Roche, Basel, Switzerland). Serum calcium was determined by colorimetric assay (lower detection limit, $0.2 \mathrm{mg} / \mathrm{dL}$; intra-assay coefficient of variation [CV], 0.9\%; inter-assay CV, 1.6\%; Roche Diagnostics, Mannheim, Germany) and serum creatinine was determined by enzymatic colorimetric assay (lower detection limit, 0.03 $\mathrm{mg} / \mathrm{dL}$; intra-assay $\mathrm{CV}, 0.9 \%$; inter-assay $\mathrm{CV}, 1.1 \%$; Roche Diagnostics). The $25(\mathrm{OH}) \mathrm{D}$ was determined by ARUP Laboratories using the DiaSorin Liaison $\mathrm{ClA}$ technology that measured both $25(\mathrm{OH}) \mathrm{D}_{2}$ and $25(\mathrm{OH}) \mathrm{D}_{3}$ (lower detection limit, $0.8 \mathrm{ng} / \mathrm{mL}$; intra-assay CV, 6.7\%; inter-assay CV, 6.3\%). The laboratory participated in regular Vitamin D External Quality Assessment Scheme performance assessments and passed quality assurance minimal standards. PTH was determined using Roche Modular E170 analyzer using an electrochemiluminescence immunoassay (lower detection 
limit, $1.2 \mathrm{pg} / \mathrm{mL}$; intra-assay CV, $1.9 \%$; inter-assay CV, 4.5\%; Roche Diagnostics). There were no changes to any of the tests used during the duration of time covered by the study. DXA scans were performed using a Hologic Discovery v12.6.2 instrument (Hologic Inc., Bedford, MA, USA; Center-specific hip and spine least significant change $[L S C]=0.020 \mathrm{~g} / \mathrm{cm}^{2}$ ). Areal BMD was measured at the total hip, femoral neck, and lumbar spine (L1-4) and expressed in grams $/ \mathrm{cm}^{2}$.

All data were extracted from the patients' EMR by 1 investigator (TK) and cross-checked by another investigator (ASR).

\section{Statistical analyses}

Statistical analyses were conducted using SAS for Windows, version 9.3 (SAS Institute Inc., Cary, NC, USA) and statistical significance was defined as a $P$-value less than 0.05 without correction for multiple testing. Due to the small sample size, data are presented as median (interquartile range) or percentage.

We began by examining patient characteristics and baseline DXA and laboratory results. We also calculated the number of doses of denosumab that each patient received and the duration of time between the last dose of denosumab and the first dose of ZA.

We then examined the change in BMD from baseline (before initiation of denosumab) to the end of the denosumab treatment course and between the end of the denosumab treatment course and the first DXA scan following ZA. We used nonparametric methods (Wilcoxon signedrank test) to compare these data points. Finally, we used univariable logistic regression to examine the association between changes in BMD and total number of denosumab doses received and between changes in BMD and the duration of time between the last dose of denosumab and the first dose of ZA. Multivariable analyses were not performed due to the small sample size. The institutional review board approved this study.

\section{RESULTS}

\section{Baseline evaluation}

We identified 12 patients who met the study inclusion and exclusion criteria. A description of our study cohort is presented in Table 1. Three of the patients had their initial DXA scan prior to initiation of denosumab performed out-
Table 1. Patient characteristics

\begin{tabular}{|c|c|}
\hline Characteristics & Value $(\mathrm{N}=12)$ \\
\hline Age (yr), median (IQR) & $76.8(8.8)$ \\
\hline Sex (female) & $12(100.0)$ \\
\hline Race (white) & $12(100.0)$ \\
\hline BMI $\left(\mathrm{kg} / \mathrm{m}^{2}\right)$, median (IQR) & $21.9(4.5)$ \\
\hline 25(OH)D (ng/mL), median (IOR) & $37.5(13.0)$ \\
\hline Calcium (mg/dL), median (IQR) & $9.8(0.7)$ \\
\hline PTH (pg/mL), median (IQR) & $40.1(3.9)$ \\
\hline Creatinine (mg/dL), median (IQR) & $0.7(0.2)$ \\
\hline \multicolumn{2}{|l|}{ Smoking } \\
\hline Current smoker & $1(8.3)$ \\
\hline Previous smoker & $4(33.3)$ \\
\hline Alcoholic drinks per week, median (IQR) & $1(1.5)$ \\
\hline \multicolumn{2}{|l|}{ Trauma } \\
\hline Presence of low-trauma fracture before Dmab & $2(16.7)$ \\
\hline Presence of low-trauma vertebral fracture before Dmab & $1(8.3)$ \\
\hline \multicolumn{2}{|l|}{$\mathrm{BMD}\left(\mathrm{gm} / \mathrm{cm}^{2}\right)$, median $(\mathrm{IQR})^{\mathrm{b})}$} \\
\hline Lumbar spine & $0.82(0.03)$ \\
\hline Total hip & $0.69(0.05)$ \\
\hline Femoral neck & $0.59(0.02)$ \\
\hline \multicolumn{2}{|l|}{ Treatment received } \\
\hline Received bisphosphonate before Dmab & $7(58.3)$ \\
\hline $\begin{array}{l}\text { Bisphosphonate washout before Dmab (yr), median } \\
(\text { (IQR) })^{c}\end{array}$ & $4(2.5)$ \\
\hline No. of Dmab doses, median (IQR) & $5.3(1.2)$ \\
\hline No. of ZA doses, median (IQR) & $2(0.3)$ \\
\hline Patients with osteoporosis at time of transition to ZA & $6(50.0)$ \\
\hline $\begin{array}{l}\text { Lag time between last dose of Dmab and first dose of } \\
\text { ZA (days), median (IOR) }\end{array}$ & $201.5(31.3)$ \\
\hline
\end{tabular}

The data is presented as median (IQR) or number (\%).

alPTH available in 5 patients. ${ }^{b}$ Baseline BMD in 9. ${ }^{\text {cl} B i s p h o s p h o n a t e ~ w a s h-~}$ out applies to 7 patients who received bisphosphonate before Dmab. IOR, interquartile range; $\mathrm{BMI}$, body mass index; $25(\mathrm{OH}) \mathrm{D}, 25$-hydroxy-vitamin D; PTH, parathyroid hormone; BMD, bone mineral density; Dmab, denosumab; ZA, zoledronic aid.

side our medical center, and those measurements were excluded from the analysis. All other DXA scans were performed in our laboratory. At the time of transitioning to consolidation therapy, 6 of the patients still had osteoporosis (defined by a T-score $\leq-2.5$ at the lumbar spine, hip or femoral neck), and 6 had osteopenia. The reasons for transition to ZA in patients who still had osteoporosis was plateauing of BMD after 5 to 6 doses of denosumab in 4 patients, and patient request due to frequent traveling in 2 patients. The average $t$-scores in all 12 patients at the lumbar spine, total hip, and femoral neck were $-1.23,-1.66$, and -1.93 , respectively. 


\section{Treatment and follow-up}

Patients received a median of 5.3 doses of denosumab 6 months apart (range, 3-7 doses). All patients subsequently received at least one dose of ZA (median, 2; range, 1-3 doses). The median duration of time between the last dose of denosumab and the first dose of ZA was 201.5 days ( 6.7 months).

All patients underwent a DXA measurement in our lab at the end of their denosumab treatment course and another DXA after the first dose of ZA. The median duration of time between the first dose of ZA and the corresponding followup DXA scan was 12.0 (1.0) months.

Five patients received a second yearly dose of ZA and had another DXA measurement following the second dose. In these patients, the median duration of time between the first and second doses of ZA was 12.7 (0.4) months. The median duration of time between the second dose of ZA and the corresponding DXA was 12.3 (0.3) months.

None of the patients suffered a clinical fracture while on denosumab. After transition to ZA, 1 patient sustained a distal radial fracture upon a fall. The fracture happened

Table 2. Changes in bone density measurements over time

\begin{tabular}{lccl}
\hline \multirow{2}{*}{\begin{tabular}{c} 
DXA measurement $\begin{array}{c}\text { DXA time } \\
\text { site }\end{array}$ \\
\cline { 2 - 4 }
\end{tabular}} & $\begin{array}{c}\text { Pre-Dmab } \\
(\mathrm{N}=9)\end{array}$ & $\begin{array}{c}\text { Post-Dmab } \\
(\mathrm{N}=12)\end{array}$ & $\begin{array}{c}\text { Post-ZA } \\
(\mathrm{N}=12)\end{array}$ \\
\hline $\mathrm{BMD}\left(\mathrm{g} / \mathrm{cm}^{2}\right)$ & & & \\
Lumbar spine & $0.82(0.03)$ & $0.90(0.15)^{\mathrm{a})}$ & $0.87(0.08)^{\mathrm{a})}$ \\
Total hip & $0.69(0.05)$ & $0.75(0.04)$ & $0.72(0.08)$ \\
Femoral neck & $0.59(0.02)$ & $0.63(0.06)^{\mathrm{b})}$ & $0.62(0.05)^{\mathrm{c})}$ \\
\hline
\end{tabular}

The data is presented as median (interquartile range).

a)Lumbar spine measurement excluded in 1 patient due to severe degenerative changes. ${ }^{\text {b) }}$ Statistically significant increase from pre-Dmab, $P=0.008$. ${ }^{c}$ Statistically significant decrease from post-Dmab, $P=0.040$. DXA, dual energy $X$-ray absorptiometry; Pre-Dmab, DXA scans prior to initiation of denosumab; Post-Dmab, DXA scans after completion of treatment with denosumab; Post-ZA, DXA scans after the first dose of zoledronic acid; BMD, bone mineral density. when she lost her balance while trying to sit on a stool and fell down on her extended wrist. She had received 3 doses of denosumab and 1 dose of $Z A$, the latter being about 6 months prior to the fracture. Her DXA scan about 6 months after the fracture showed a T score of -1.9 at the lumbar spine, -1.0 at the total hip, and -1.6 at the femoral neck. While spine X-rays were not routinely obtained on all patients, 3 patients underwent spine imaging after switching from denosumab to ZA ( 2 patients underwent thoracic and lumbar spine imaging, and 1 patient underwent lumbar spine imaging only), and no vertebral fractures were identified.

\section{Changes in BMD over time}

Table 2 shows the mean BMD for all patients with available DXA scans prior to initiation of denosumab (pre-Dmab, $\mathrm{N}=9$ ), after completion of treatment with denosumab (postDmab, $N=12$ ) and after the first dose of $Z A$ (post- $Z A, N=12$ ). As mentioned previously, the analyses excluded 3 pre-denosumab DXA scans that were performed outside our medical center.

When comparing the changes in measurements between the pre-Dmab and that obtained at the time of completion of denosumab, a statistically significant increase in BMD $(P=0.008)$ was seen at the femoral neck. A non-significant increase was noted at the lumbar spine and total hip.

When comparing the DXA scan obtained at the end of denosumab treatment and that obtained after the first dose of $Z A$, we noted a significant decline in BMD $(P=0.04)$ at the femoral neck. A non-significant downward trend was seen at the lumbar spine and total hip. The BMD at all 3 sites however remained higher than the BMD measured prior to initiation of denosumab. Patients who experienced a decline in BMD with ZA underwent a limited evaluation for secondary causes of bone loss (including abnormal renal function, vitamin D deficiency, hyperthyroidism, and

Table 3. Relative changes in BMD upon switching from Dmab to ZA

\begin{tabular}{|c|c|c|c|c|c|}
\hline \multirow{2}{*}{ Site } & \multicolumn{3}{|c|}{ All patients } & \multirow{2}{*}{$\begin{array}{l}\text { Patients with osteopenia } \\
\text { at end of Dmab } \\
\text { Percent of median BMD } \\
\text { gain that is lost with ZA }\end{array}$} & \multirow{2}{*}{$\begin{array}{l}\text { Patients with osteopo- } \\
\text { rosis at end of Dmab } \\
\text { Percent of median BMD } \\
\text { gain that is lost with ZA }\end{array}$} \\
\hline & $\begin{array}{l}\text { Median BMD gain on } \\
\text { Dmab }\left(\mathrm{g} / \mathrm{cm}^{2}\right)\end{array}$ & $\begin{array}{l}\text { Median BMD loss after } \\
\text { switching to ZA }\left(\mathrm{g} / \mathrm{cm}^{2}\right)\end{array}$ & $\begin{array}{l}\text { Percent of median BMD } \\
\text { gain that is lost with ZA }\end{array}$ & & \\
\hline Lumbar spine & 0.048 & 0.011 & $23 \%$ & $7 \%$ & $61 \%$ \\
\hline Total hip & 0.034 & 0.016 & $47 \%$ & $43 \%$ & $220 \%$ \\
\hline
\end{tabular}

BMD, bone mineral density; Dmab, denosumab; ZA, zoledronic aid. 

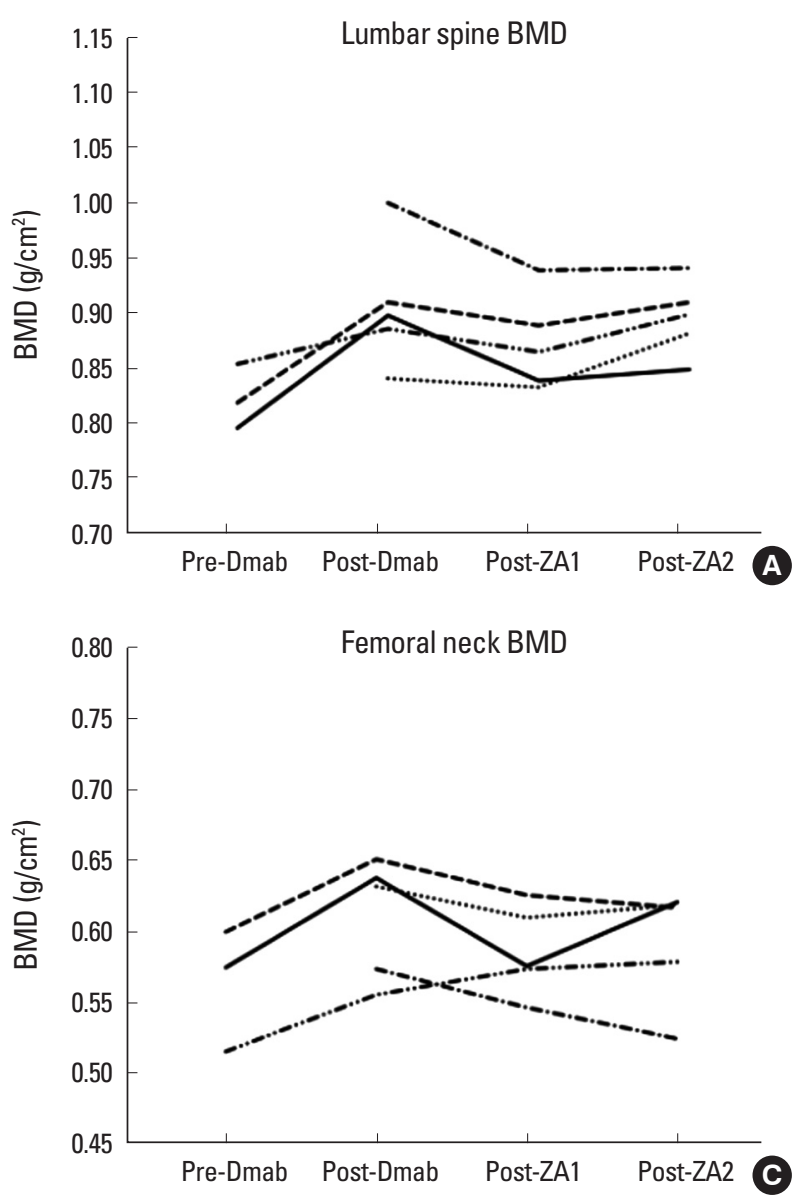

hyperparathyroidism), and none was found.

On average, within approximately 1 year of switching from denosumab to $Z A$, patients lost $23 \%$ of the BMD gain they had accrued at the lumbar spine while on denosumab, $47 \%$ of the BMD gain accrued at the total hip and $54 \%$ of the BMD gain accrued at the femoral neck (Table 3). Of the patients who had osteopenia at the end of denosumab, 4 (67\%) experienced some loss in BMD in at least one site. Of the patients with osteoporosis at the end of denosumab $5(83 \%)$ experienced some loss of BMD in at least one site. Table 3 shows the proportion of BMD gain lost upon switching to ZA in each of these subgroups.

In a univariable logistic regression, the decline in BMD between the DXA scan obtained at the end of denosumab and the one obtained after the first dose of ZA was independent of the number of denosumab doses (for change in $B M D$ at the lumbar spine $O R=0.48$ and $P=0.22$; at the total hip $\mathrm{OR}=0.70$ and $P=0.55$; and at the femoral neck $\mathrm{OR}=0.70$ and $P=0.55$ ) It was also independent of the time between the last dose of denosumab and the first dose of

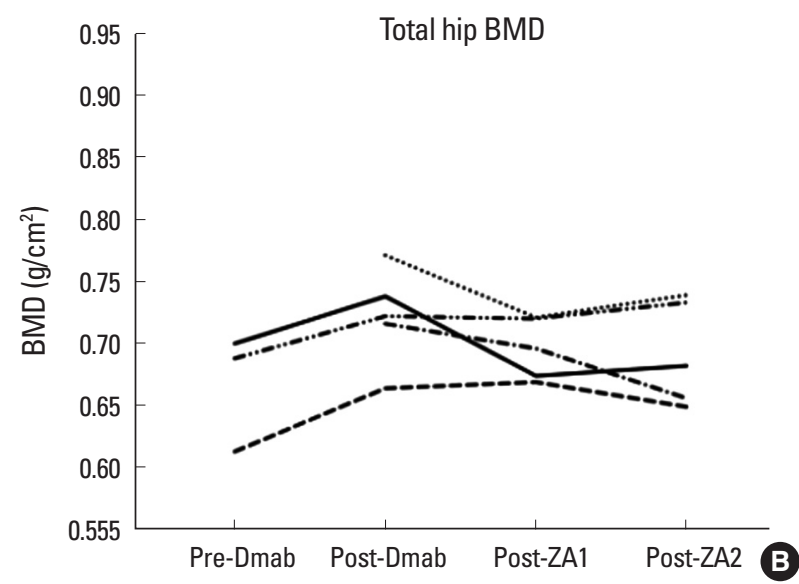

Fig. 1. Bone mineral density (BMD) and T-score measurements in 5 patients who received 2 doses of zoledronic acid. Each line represents an individual patient. DXA, dual energy X-ray absorptiometry; Pre-Dmab, DXA scans prior to initiation of denosumab; Post-Dmab, DXA scans after completion of treatment with denosumab; Post-ZA1, DXA scans after the first dose of zoledronic acid; Post-ZA2, DXA scans after the second dose of zoledronic acid.

$\mathrm{ZA}$ (for change in $\mathrm{BMD}$ at the lumbar spine $\mathrm{OR}=1.0$ and $P=0.54$; at the total hip $\mathrm{OR}=1.0$ and $P=0.54$; and the femoral neck $\mathrm{OR}=1.0$ and $P=0.3$ )

Five individuals received a second dose of $Z A$ and had another DXA scan 1 year later. We examined the changes in BMD between the DXA that followed the first ZA dose and the one following the second dose. Fig. 1 shows these changes for the individual patients with available data $(\mathrm{N}=5)$. The graphs suggest general stabilization of BMD at the lumbar spine, total hip, and femoral neck a year after the second annual consolidation dose of ZA. Due to the small number of patients in this subgroup, no statistical analyses on the trends were performed.

\section{DISCUSSION}

This case series suggests that patients who receive 1 dose of ZA after treatment with denosumab lose some of the BMD accrued while on denosumab. These results are consistent with previous studies where BMD loss was ob- 
served after a single consolidation dose of ZA.[15-17] The studies by Lehmann and Aeberli [16] and Reid et al. [17] measured BMD 2 to 2.5 years after ZA and could not determine the exact timeline of bone loss. However, our observations confirm what the work of Everts-Graber et al. [15] suggests, in that much of the observed bone loss happens during the first 12 months that follow the ZA infusion. In addition, our observations suggest that the bone loss might slow down when another yearly dose of ZA is given, but caution should be applied in interpreting these findings given the small sample size.

Our findings also suggest that the bone loss at the total hip and spine is much larger in patients who remained osteoporotic while on denosumab. This finding might present an argument in favor of ensuring patients' $B M D$ is in the osteopenia range on denosumab before switching them to ZA. It is worth making a note here of a recent study by Anastasilakis that randomized 57 women previously treated with denosumab for osteoporosis to another 2 doses of denosumab versus a single dose of ZA. Twelve months after randomization, an equal but non-significant increase in the lumbar spine and femoral neck BMD was seen in both groups, despite higher levels of bone turnover markers (BTMs) in the group randomized to ZA.[18] While these results contradict the decline in $B M D$ with the transition from denosumab to ZA observed by other studies, it should be noted that all patients had osteopenia at the time of transition, which might again argue in favor of attaining osteopenia with denosumab before initiation of consolidation therapy.

One possible explanation for the observed bone loss when switching from denosumab to ZA lies in the fact that bisphosphonates need "open bone surfaces" to adhere to in order to exert their antiresorptive action.[3] Since bone turnover is still profoundly suppressed 6 months after a dose of denosumab, it is likely that ZA adherence to bone surfaces is reduced when given at that time. Thus, a single dose of ZA given after completion of denosumab treatment may be partially "wasted" and not fully taken up to the bone surfaces. One would then expect a more frequently dosed bisphosphonate to have more opportunities for adherence as the bone turnover is gradually restored after denosum$a b$, thus allowing more antiresorptive action. This was indeed shown by Freemantle et al. in the DAPS study where 1 year of alendronate following 1 year of denosumab pre- vented post-denosumab bone loss.[19]

Alternatively, it may be attractive to delay ZA by more than 6 months after the last dose of denosumab to allow for some recovery of bone turnover and for the drug to adhere better to bone surfaces. Horne et al. [20] tested this hypothesis in a group of postmenopausal women treated with 1 year of romosozumab followed by 2 years of denosumab. Following denosumab, the subjects received a single dose of ZA, but the administration of ZA was delayed by an average of 65 days (i.e., it was administered about 8 months rather than 6 months after the last dose of denosumab). The bone loss 1 year after ZA was milder with this regimen.[20] While this may suggest that a delay in the timing of ZA after denosumab could enhance the antiresorptive efficacy of the drug, this approach might be associated with an increased risk of vertebral fractures, as such fractures have been observed as early as 2 months after discontinuation of denosumab.[11] Additionally, this study added the new variable of romosozumab treatment prior to denosumab, and it is not clear to what degree a change in bone quality from romosozumab may have contributed to the milder bone loss observed after ZA.

It is important to acknowledge potential limitations of this work. First, our sample size was small and this may explain the lack of statistical significance in some of our analyses. Notably however, while our analyses of BMD changes at the lumbar spine and hip did not reach statistical significance, the trends were comparable to previous studies that did achieve statistical significance.[15,16] Measurement of BTMs is also missing from our study, somewhat limiting our interpretation of the results. Finally, while our study focuses on the changes in BMD over time, it is important to acknowledge that fractures are the clinicallyrelevant outcome of low BMD. Although all clinical fractures were documented in our patients' records, routine $x$ rays to assess for subclinical fractures were not obtained. Nonetheless, there was no evidence of an increased risk of clinical vertebral fractures in our patients despite the observed decline in BMD.

In conclusion, a single dose of ZA given approximately 6 months after long-term denosumab therapy leads to a loss of a portion of the BMD gained with denosumab, and much of this loss seems to occur within 1 year of ZA, particularly in patients who are osteoporotic at the time of transition. Furthermore, limited data suggest that the loss in BMD may 
plateau after a second dose of ZA. However, larger and longer-term studies with careful evaluation of fracture risk are needed to provide a definitive answer on the safety and best approach to post-denosumab consolidation therapy with ZA.

\section{DECLARATIONS}

\section{Funding}

The authors received no financial support for this article.

\section{Ethics approval and consent to participate}

The study was reviewed and approved by the Institutional Review Board for human subjects' research.

\section{Conflict of interest}

No potential conflict of interest relevant to this article was reported.

\section{ORCID}

Amal Shibli-Rahhal https://orcid.org/0000-0003-2405-8963

\section{REFERENCES}

1. Lewiecki EM, Bilezikian JP. Denosumab for the treatment of osteoporosis and cancer-related conditions. Clin Pharmacol Ther 2012;91:123-33. http://dx.doi.org/10.1038/ clpt.2011.268.

2. Cummings SR, San Martin J, McClung MR, et al. Denosumab for prevention of fractures in postmenopausal women with osteoporosis. N Engl J Med 2009;361:756-65. http:// dx.doi.org/10.1056/NEJMoa0809493.

3. Bone HG, Chapurlat R, Brandi ML, et al. The effect of three or six years of denosumab exposure in women with postmenopausal osteoporosis: results from the FREEDOM extension. J Clin Endocrinol Metab 2013;98:4483-92. http:// dx.doi.org/10.1210/jc.2013-1597.

4. Bone HG, Bolognese MA, Yuen CK, et al. Effects of denosumab treatment and discontinuation on bone mineral density and bone turnover markers in postmenopausal women with low bone mass. J Clin Endocrinol Metab 2011; 96:972-80. http://dx.doi.org/10.1210/jc.2010-1502.

5. Miller PD, Bolognese MA, Lewiecki EM, et al. Effect of denosumab on bone density and turnover in postmenopausal women with low bone mass after long-term continued, discontinued, and restarting of therapy: a randomized blinded phase 2 clinical trial. Bone 2008;43:222-9. http:// dx.doi.org/10.1016/j.bone.2008.04.007.

6. Leder BZ, Tsai JN, Jiang LA, et al. Importance of prompt antiresorptive therapy in postmenopausal women discontinuing teriparatide or denosumab: The Denosumab and Teriparatide Follow-up study (DATA-Follow-up). Bone 2017;98:54-8. http://dx.doi.org/10.1016/j.bone.2017.03.006.

7. Florez H, Ramírez J, Monegal A, et al. Spontaneous vertebral fractures after denosumab discontinuation: A case collection and review of the literature. Semin Arthritis Rheum 2019;49:197-203. http://dx.doi.org/10.1016/j. semarthrit.2019.02.007.

8. Popp AW, Zysset PK, Lippuner K. Rebound-associated vertebral fractures after discontinuation of denosumab-from clinic and biomechanics. Osteoporos Int 2016;27:1917-21. http://dx.doi.org/10.1007/s00198-015-3458-6.

9. Lamy O, Gonzalez-Rodriguez E, Stoll D, et al. Severe rebound-associated vertebral fractures after denosumab discontinuation: 9 clinical cases report. J Clin Endocrinol Metab 2017;102:354-8. http://dx.doi.org/10.1210/jc.20163170.

10. Aubry-Rozier B, Gonzalez-Rodriguez E, Stoll D, et al. Severe spontaneous vertebral fractures after denosumab discontinuation: three case reports. Osteoporos Int 2016; 27:1923-5. http://dx.doi.org/10.1007/s00198-015-3380-y.

11. Anastasilakis AD, Polyzos SA, Makras P, et al. Clinical features of 24 patients with rebound-associated vertebral fractures after denosumab discontinuation: Systematic review and additional cases. J Bone Miner Res 2017;32: 1291-6. http://dx.doi.org/10.1002/jbmr.3110.

12. Anastasilakis AD, Makras P. Multiple clinical vertebral fractures following denosumab discontinuation. Osteoporos Int 2016;27:1929-30. http://dx.doi.org/10.1007/s00198015-3459-5.

13. Cummings SR, Ferrari $S$, Eastell R, et al. Vertebral fractures after discontinuation of denosumab: A post hoc analysis of the randomized placebo-controlled FREEDOM trial and its extension. J Bone Miner Res 2018;33:190-8. http://dx. doi.org/10.1002/jbmr.3337.

14. McClung MR, Wagman RB, Miller PD, et al. Observations following discontinuation of long-term denosumab therapy. Osteoporos Int 2017;28:1723-32. http://dx.doi.org/ 10.1007/s00198-017-3919-1.

15. Everts-Graber J, Reichenbach S, Ziswiler HR, et al. A single 
infusion of zoledronate in postmenopausal women following denosumab discontinuation results in partial conservation of bone mass gains. J Bone Miner Res 2020;35: 1207-15. http://dx.doi.org/10.1002/jbmr.3962.

16. Lehmann T, Aeberli D. Possible protective effect of switching from denosumab to zoledronic acid on vertebral fractures. Osteoporos Int 2017;28:3067-8. http://dx.doi.org/ 10.1007/s00198-017-4108-y.

17. Reid IR, Horne AM, Mihov B, et al. Bone loss after denosumab: Only partial protection with zoledronate. Calcif Tissue Int 2017;101:371-4. http://dx.doi.org/10.1007/s00223017-0288-x.

18. Anastasilakis AD, Papapoulos SE, Polyzos SA, et al. Zoledronate for the prevention of bone loss in women discon- tinuing denosumab treatment. A prospective 2-year clinical trial. J Bone Miner Res 2019;34:2220-8. http://dx.doi. org/10.1002/jbmr.3853.

19. Freemantle N, Satram-Hoang S, Tang ET, et al. Final results of the DAPS (Denosumab Adherence Preference Satisfaction) study: a 24-month, randomized, crossover comparison with alendronate in postmenopausal women. Osteoporos Int 2012;23:317-26. http://dx.doi.org/10.1007/s00198011-1780-1.

20. Horne AM, Mihov B, Reid IR. Bone loss after romosozumab/denosumab: Effects of bisphosphonates. Calcif Tissue Int 2018;103:55-61. http://dx.doi.org/10.1007/s00223-0180404-6. 